\title{
'Oh Bulan' Pilot Project: Sanitary Pad Usage and School Absenteeism During Menstruation Among Adolescent Girls in Rural Kelantan
}

\author{
Nur Aizati Athirah Daud ${ }^{1}$, Veronica G.K. Williams, Zuraidah Daut, Zaharah \\ Sulaiman $^{2}$ \\ ${ }^{1}$ Discipline of Clinical Pharmacy, School of Pharmaceutical Sciences, Universiti Sains Malaysia, 11800 \\ USM, Penang, Malaysia, ${ }^{2}$ Womens Health Development Unit, School of Medical Sciences, Health \\ Campus, Universiti Sains Malaysia, 16150 Kubang Kerian, Kelantan, Malaysia
}

\begin{abstract}
Introduction: Menstruation is the sign of menarche for female adolescents. Previous finding had reported a need for education to improve the knowledge on menstrual hygiene among adolescent girls living in rural Kelantan. However, the access of sanitary pads and the effect of menstruation on school attendance among this population have not been reported elsewhere.
\end{abstract}

Objectives: To determine the prevalence of sanitary pads usage and school absenteeism among adolescent girls in rural Kelantan.

Methods: This is a cross-sectional study conducted among female students of Sekolah Kebangsaan Kampung Dangar in Pasir Mas, Kelantan between February and March 2019. Data were collected using a structured questionnaire in Bahasa Malaysia, which were administered by the school counselor.

Results: A total of 172 respondents filled the questionnaires, with a mean age of 15 years (s.d. 1.4 , range 13 to 17$)$. Nearly half of them $(n=78,45.3 \%)$ are the daughters of laborers. Majority of them have fathers and mothers with the highest education level of SPM/STPM, while 7\% have fathers with no proper schooling. Their mean age of menarche is 12 . Majority of them claimed to use disposable sanitary pad $(n=148,87.2 \%)$ and would be wearing it every time during menstruation ( $\mathrm{n}=148,86.0 \%)$, while $4.7 \%$ used cloth pad. 40 (23.3\%) of respondents would only stay at home during menstruation, mainly because they felt uncomfortable $(5.8 \%)$ or having period pain $(4.1 \%) .25(14.5 \%)$ of the respondents used to be absent from school during menstruation, mainly due to period pain (12.2\%).

Conclusion: Disposable sanitary pad usage is highly prevalent among adolescents in this rural area, while menstruation have caused some of them to be absent from school.

Keywords: menstruation, menstrual hygiene, school absenteeism, adolescent health 